\title{
The effect of terminal substituents on crystal structure, mesophase behaviour and optical property of azo-ester linked materials
}

Md. Rabiul Karim ${ }^{\mathrm{a}}$, Md. Rezaul Karim Sheikh ${ }^{\mathrm{b}}$, Rosiyah Yahya ${ }^{\mathrm{c}^{*}}$, Lo Kong Mun ${ }^{\mathrm{c}}$, Noordini M. Salleh ${ }^{\mathrm{c}}$ and H.N.M Ekramul Mahmud ${ }^{\mathrm{c}}$

a Department of Chemistry, University of Rajshahi, Rajshahi 6205, Bangladesh

${ }^{\mathrm{b} D e p a r t m e n t}$ of Applied Chemistry and Chemical Engineering, University of Rajshahi, Rajshahi 6205, Bangladesh

${ }^{\mathrm{c}}$ Department of Chemistry, University of Malaya, Kuala Lumpur 50603, Malaysia

Table SI1: FT-IR vibrational frequencies for main functional groups and ${ }^{\mathbf{1}} \mathbf{H}$ NMR chemical shifts of different type of protons present in compounds C1-C6

\begin{tabular}{cccccc}
\hline \multicolumn{7}{c}{ Wavenumber $\left(\mathrm{cm}^{-1}\right)$} \\
\hline Functional groups & $(\mathrm{C}-\mathrm{H}$, aliphatic $)$ & $(\mathrm{C}=\mathrm{O}$ in ester $)$ & $(\mathrm{C}=\mathrm{C}$, vinyl $)$ & $(\mathrm{C}=\mathrm{C}$, aromatic $)$ & $(-\mathrm{N}=\mathrm{N}-)$ \\
\hline C1 & 2945,2880 & 1725 & 1634 & 1603,1583 & 1505,1471 \\
C2 & 2945,2874 & 1729 & 1638 & 1606,1573 & 1506,1489 \\
C3 & 2944,2871 & 1736 & 1637 & 1602,1583 & 1503,1486 \\
C4 & 2956,2881 & 1717 & 1632 & 1605,1584 & 1507,1473 \\
C5 & 2957,2873 & 1722 & 1642 & 1605,1584 & 1505,1473 \\
C6 & 2946,2872 & 1724 & 1637 & 1604,1596 & 1508,1476 \\
\hline
\end{tabular}

\begin{tabular}{cccccccccc}
\hline \multicolumn{7}{c}{ Chemical Shift (ppm) } \\
\hline $\begin{array}{c}\text { Type of } \\
\text { protons }\end{array}$ & $\mathrm{Ar}-\underline{\mathrm{H}}$ & $-\mathrm{CH}=\underline{\mathrm{H}}_{2}$ & $-\mathrm{C} \underline{\mathrm{H}}=\mathrm{CH}_{2}$ & $-\mathrm{CH}=\underline{\mathrm{H}}_{2}$ & $\mathrm{Ar}-\mathrm{OC} \underline{\mathrm{H}}_{2}-$ & $-\underline{\mathrm{CH}}_{2}-\mathrm{OCO}-$ & $-\mathrm{OC} \underline{\mathrm{H}}_{3}$ & $-\mathrm{C} \underline{\mathrm{H}}_{2}-$ \\
\hline C1 & $8.33-6.98$ & $6.43-6.37$ & $6.16-6.06$ & $5.84-5.79$ & $4.21-4.15$ & $4.09-4.02$ & - & $1.89-1.41$ \\
C2 & $8.33-6.98$ & $6.43-6.37$ & $6.16-6.08$ & $5.85-5.79$ & $4.21-4.15$ & $4.09-4.02$ & - & $1.89-1.41$ \\
C3 & $8.34-6.98$ & $6.43-6.37$ & $6.16-6.08$ & $5.84-5.79$ & $4.22-4.15$ & $4.09-4.02$ & - & $1.90-1.42$ \\
C4 & $8.32-6.90$ & $6.42-6.35$ & $6.15-6.06$ & $5.83-5.77$ & $4.19-4.14$ & $4.07-4.01$ & $3.82-3.78$ & $1.88-1.42$ \\
C5 & $8.35-6.90$ & $6.42-6.40$ & $6.15-6.07$ & $5.84-5.78$ & $4.22-4.14$ & $4.09-4.00$ & - & $1.89-1.39$ \\
C6 & $8.32-6.90$ & $6.42-6.40$ & $6.16-6.07$ & $5.88-5.78$ & $4.20-4.14$ & $3.95-3.89$ & - & $1.88-1.00$ \\
\hline
\end{tabular}


Table SI2: Selected bond lengths $(\AA)$ and bond angles $\left({ }^{\circ}\right)$ of compounds C1, C4 and C5

\begin{tabular}{|c|c|c|c|c|c|c|c|}
\hline Bond lengths $(\AA)$ & $\mathrm{C1}$ & $\mathrm{C4}$ & $\mathbf{C 5}$ & Bond angles $\left(^{\circ}\right)$ & $\mathrm{C1}$ & $\mathrm{C4}$ & $\mathrm{C5}$ \\
\hline $\mathrm{F}(1)-\mathrm{C}(26)$ & 1.361(3) & - & - & $\mathrm{F}(1)-\mathrm{C}(26)-\mathrm{C}(25)$ & $118.6(2)$ & - & - \\
\hline $\mathrm{N}(1)-\mathrm{N}(2)$ & $1.256(2)$ & $1.2603(18)$ & $1.2506(19)$ & $\mathrm{N}(2)-\mathrm{N}(1)-\mathrm{C}(13)$ & $113.52(18)$ & $114.23(13)$ & $112.81(15)$ \\
\hline $\mathrm{N}(1)-\mathrm{C}(13)$ & $1.419(3)$ & $1.4149(19)$ & $1.420(2)$ & $\mathrm{N}(1)-\mathrm{N}(2)-\mathrm{C}(16)$ & $114.84(18)$ & $113.33(13)$ & $116.16(15)$ \\
\hline $\mathrm{N}(2)-\mathrm{C}(16)$ & $1.423(3)$ & $1.4278(19)$ & $1.418(2)$ & $\mathrm{C}(3)-\mathrm{O}(2)-\mathrm{C}(4)$ & 115.19(18) & $117.35(12)$ & 116.81(14) \\
\hline $\mathrm{O}(1)-\mathrm{C}(3)$ & $1.205(3)$ & $1.2075(18)$ & $1.198(2)$ & $\mathrm{C}(10)-\mathrm{O}(3)-\mathrm{C}(9)$ & $117.94(17)$ & $118.32(12)$ & $120.24(14)$ \\
\hline $\mathrm{O}(2)-\mathrm{C}(3)$ & $1.344(3)$ & $1.3399(18)$ & $1.330(2)$ & $\mathrm{C}(22)-\mathrm{O}(5)-\mathrm{C}(23)$ & $119.19(16)$ & $120.98(12)$ & $122.57(15)$ \\
\hline $\mathrm{O}(3)-\mathrm{C}(10)$ & $1.361(3)$ & $1.3587(18)$ & $1.359(2)$ & $\mathrm{C}(1)-\mathrm{C}(2)-\mathrm{C}(3)$ & $121.5(2)$ & $120.93(15)$ & $122.8(2)$ \\
\hline $\mathrm{O}(4)-\mathrm{C}(22)$ & $1.205(2)$ & $1.2015(19)$ & $1.194(2)$ & $\mathrm{H}(1 \mathrm{~A})-\mathrm{C}(1)-\mathrm{H}(1 \mathrm{~B})$ & 120.0 & 120.0 & 120.0 \\
\hline $\mathrm{O}(5)-\mathrm{C}(22)$ & $1.363(2)$ & $1.3543(19)$ & $1.352(2)$ & $\mathrm{O}(1)-\mathrm{C}(3)-\mathrm{O}(2)$ & $123.6(2)$ & $123.43(14)$ & $124.53(19)$ \\
\hline $\mathrm{O}(6)-\mathrm{C}(29)$ & - & $1.428(2)$ & $1.427(2)$ & $\mathrm{O}(2)-\mathrm{C}(4)-\mathrm{H}(4 \mathrm{~B})$ & 109.9 & 110.5 & 110.1 \\
\hline $\mathrm{C}(1)-\mathrm{H}(1 \mathrm{~A})$ & 0.9300 & 0.9300 & 0.9300 & $C(4)-C(5)-C(6)$ & $111.0(2)$ & $113.32(13)$ & $112.35(16)$ \\
\hline $\mathrm{C}(1)-\mathrm{H}(1 \mathrm{~B})$ & 0.9300 & 0.9300 & 0.9300 & $\mathrm{O}(3)-\mathrm{C}(9)-\mathrm{C}(8)$ & $108.10(18)$ & $107.44(12)$ & $107.05(15)$ \\
\hline$C(2)-C(3)$ & $1.478(3)$ & $1.480(2)$ & $1.459(3)$ & $\mathrm{C}(13)-\mathrm{C}(12)-\mathrm{C}(11)$ & $120.9(2)$ & 119.3 & $120.57(17)$ \\
\hline $\mathrm{C}(4)-\mathrm{H}(4 \mathrm{~A})$ & 0.9700 & 0.9700 & 0.9700 & $\mathrm{C}(12)-\mathrm{C}(13)-\mathrm{N}(1)$ & $116.75(19)$ & $116.29(14)$ & $123.61(16)$ \\
\hline$C(5)-(6)$ & $1.520(3)$ & $1.520(2)$ & $1.510(2)$ & $\mathrm{C}(17)-\mathrm{C}(16)-\mathrm{N}(2)$ & $124.94(19)$ & $124.24(14)$ & $114.55(16)$ \\
\hline $\mathrm{C}(11)-\mathrm{H}(11)$ & 0.9300 & 0.9300 & 0.9300 & $\mathrm{C}(21)-\mathrm{C}(16)-\mathrm{N}(2)$ & $115.19(19)$ & $115.86(13)$ & $126.14(16)$ \\
\hline$C(14)-C(15)$ & $1.371(3)$ & 1.371(3) & $1.385(2)$ & $C(21)-C(20)-C(19)$ & $120.39(19)$ & $119.81(14)$ & $121.01(17)$ \\
\hline $\mathrm{C}(18)-\mathrm{H}(18)$ & 0.9300 & 0.9300 & 0.9300 & $\mathrm{C}(28)-\mathrm{C}(23)-\mathrm{O}(5)$ & $121.8(2)$ & $115.49(13)$ & $125.33(18)$ \\
\hline $\mathrm{C}(19)-\mathrm{C}(22)$ & $1.478(3)$ & $1.485(2)$ & $1.476(3)$ & $\mathrm{C}(23)-\mathrm{C}(28)-\mathrm{C}(27)$ & $118.7(2)$ & $120.12(14)$ & $120.16(19)$ \\
\hline$C(23)-C(24)$ & $1.378(3)$ & $1.391(2)$ & $1.372(3)$ & $\mathrm{O}(6)-\mathrm{C}(26)-\mathrm{C}(27)$ & - & $125.02(15)$ & $125.01(18)$ \\
\hline$C(26)-C(27)$ & $1.374(3)$ & $1.387(2)$ & $1.372(3)$ & $\mathrm{C}(29)-\mathrm{C}(30)-\mathrm{H}(30 \mathrm{~B})$ & - & - & 109.5 \\
\hline$C(29)-C(30)$ & - & - & $1.492(3)$ & & & & \\
\hline
\end{tabular}

Table SI3: Thermal analysis data for compounds C1- C6

\begin{tabular}{lcccccc}
\hline & $\begin{array}{c}\mathrm{T}_{\mathrm{d}(5 \%)} \\
\left({ }^{\circ} \mathrm{C}\right)\end{array}$ & \multicolumn{2}{c}{$1^{\text {st }}$ decomposition } & \multicolumn{2}{c}{$2^{\text {nd }}$ decomposition } & \multicolumn{2}{c}{ Char yield } \\
& & & & & \\
& & & & \\
& & & & \\
& & Wt. loss $(\%)$ & Temp. $\left({ }^{\circ} \mathrm{C}\right)$ & Wt. loss $(\%)$ & \\
\hline C1 & 316 & $271-392$ & 36 & $392-556$ & 35 & 23 \\
C2 & 318 & $264-435$ & 48 & $435-543$ & 21 & 20 \\
C3 & 317 & $284-389$ & 51 & $403-474$ & 13 & 16 \\
C4 & 318 & $266-352$ & 38 & $352-536$ & 33 & 24 \\
C5 & 317 & $265-391$ & 40 & $391-535$ & 32 & 18 \\
C6 & 319 & $266-400$ & 43 & $400-525$ & 27 & 15 \\
\hline
\end{tabular}




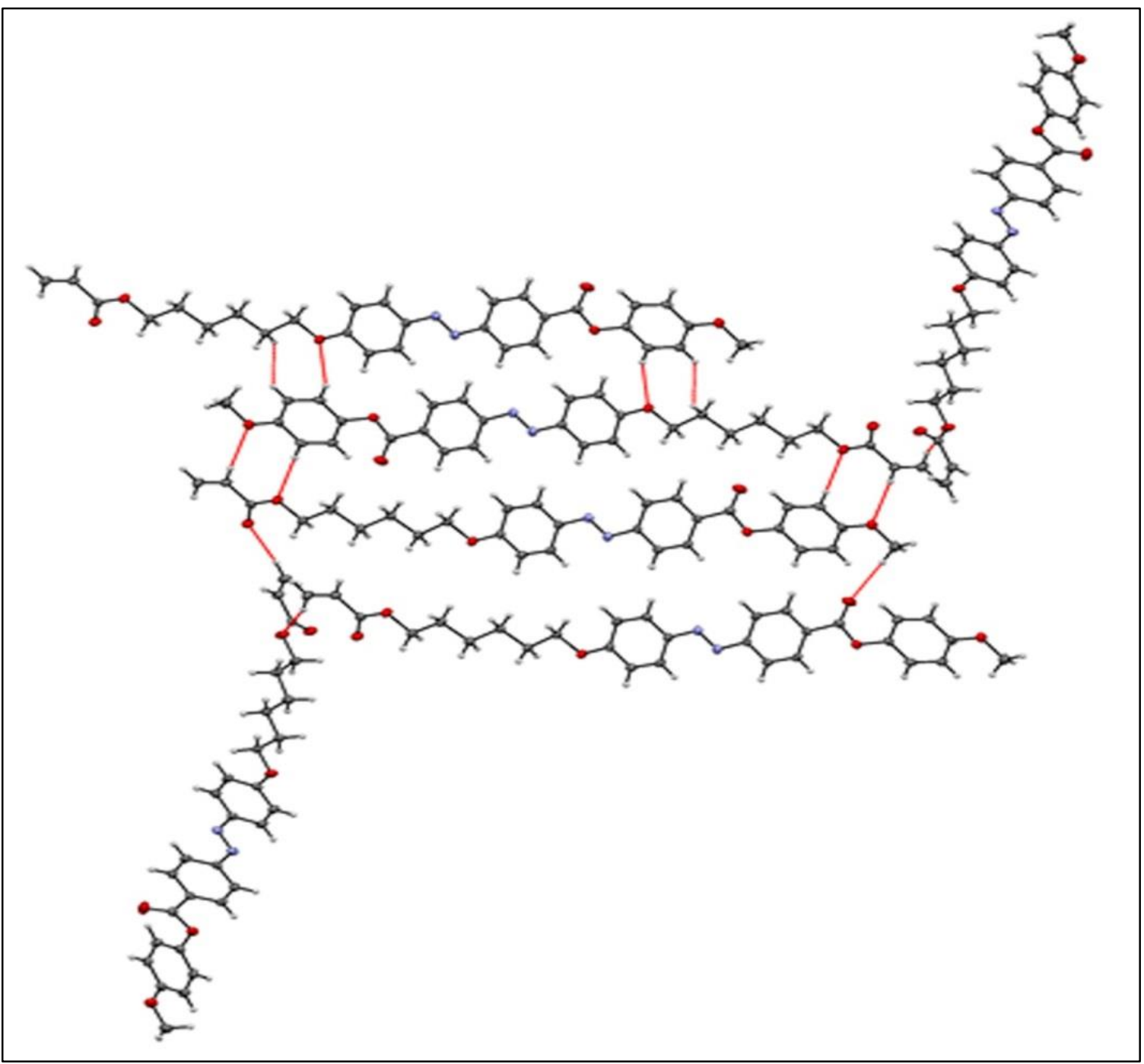

Figure SI1: Crystal structure of compound $\mathbf{C 4}$ with the hydrogen bond motifs

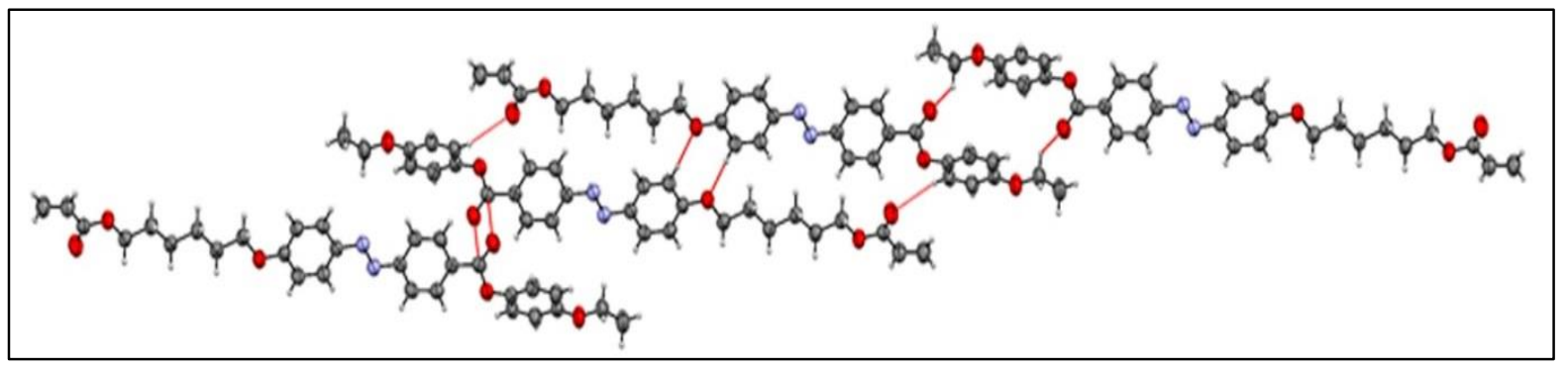

Figure SI2: Crystal structure of compound C5 with the hydrogen bond motifs 